\title{
Изобилие классов топологической сопряженно- сти для блуждающего множества внутренних отображений
}

\section{Игорь Юрьевич Власенко}

Аннотация Для гомеоморфизмов поверхностей с точностью до топологического сопряжения существует только одна двусвязная неподвижная регулярная компонента блуждающего множества. Однако для необратимых внутренних отображений это не так. В работе построен пример бесконечной последовательности внутренних отображений сферы с инвариантной двусвязной регулярной компонентой, таких, что эти отображения в сужении на свою регулярную компоненту попарно топологически не сопряжены.

Ключевые слова внутренние отображения, классы топологической сопряженности, блуждающее множество

\section{УДК 517.938 .5}

Пусть $S^{2}$ - двумерная сфера и $f: S^{2} \rightarrow S^{2}$ - ее внутреннее отображение, т. е. непрерывный открытый (образ любого открытого множества открыт) конечнократный (у каждой точки число прообразов конечно) эпиморфизм. Подробнее о внутренних отображениях см. [7].

В работах [1], [2] Биркгофом и Смитом было показано, что для гомеоморфизмов поверхностей любая периодическая регулярная компонента $S$ блуждающего множества имеет топологический тип либо односвязной либо двусвязной области в $\mathbb{R}^{2}$, причем в случае двусвязной области обе компоненты связности границы $S$ лежат во множестве неблуждающих точек. Для каждого из этих типов компонент в сужении на траекторию такой компо- 
ненты гомеоморфизмы топологически сопряжены ${ }^{1}$ : это закрывает вопрос о классификации регулярных компонент гомеоморфизмов. В [3] эти исследования были продолжены на случай больших размерностей.

Таким образом, в случае гомеоморфизмов динамика на множестве блуждающих точек устроена относительно просто. Например, для гомеоморфизмов сферы, у которых неблуждающее множество состоит из двух точек стока и источника, ограничения этих гомеоморфизмов на блуждающее множество принадлежат одному классу топологической сопряженности.

Однако, как здесь показано, для необратимых внутренних отображений ситуация существенно отличается. Уже в случае разветвленного накрывающего внутреннего отображения сферы, у которого неблуждающее множество состоит из двух точек - стока и источника, на блуждающем множестве существует бесконечное множество классов топологической сопряженности.

Для доказательства этого утверждения в работе построен пример счетного семейства попарно топологически различных внутренних отображений двумерной сферы $S^{2}$ указанного выше типа.

Отдельную благодарность хочу выразить рецензенту статьи за выявленные ошибки в первом варианте примера.

\section{Предварительные сведения.}

Обозначим через $O_{f}^{+}(x)$ положительную полутраекторию точки $x$, т. е. множество $\left\{f^{n}(x) \mid n \geq 0\right\}$. Обозначим через $O_{f}^{-}(x)$ отрицательную полутраекторию точки $x$, т. е. множество $\left\{f^{n}(x) \mid n<0\right\}$. Широкой траекторией $O_{f}(x)$ точки $x$ назовем множество $\cup_{y \in O_{f}^{+}(x)} O_{f}^{-}(y)$.

Так как $f$ - конечнократный эпиморфизм, то естественно эти траектории воспринимать как наборы из отдельных точек.

В отличие от гомеоморфизмов, для которых траектория точки в точности состоит из ее положительной и отрицательной полутраекторий, у внутренних отображений широкая траектория точки имеет и другие точки. Введем еще одно естественное подмножество широкой траектории точки, которое не нигде не пересекается с ее положительной и отрицательной полутраекториями, кроме как в самой точке.

Определение 1 Нейтралъным сечением траектории точки $x$ назовем множество $\left\{f^{-n}\left(f^{n}(x)\right) \mid n \geq 0\right\}$. Обозначим ее через $O_{f}^{\perp}(x)$.

Как легко видеть из определения, если среди образов $x$ нет периодической точки, a $f$ имеет в точках орбиты больше одного прообраза, то широкая

$1 f$ и $g$ топологически сопряжены, если $\exists$ гомеоморфизм $h: f h=h g$. Подробнее см. [5]. 
траектория точки $x$ распадается на бесконечное число нейтральных сечений, при чем каждое нейтральное сечение состоит из бесконечного числа точек.

Определение 2 Точка $x$ называется блуждающей точкой $f$, если найдется такая ее окрестность $U$, что $f^{m}(U) \cap U=\emptyset$ для всех $m \in \mathbb{Z}$.

Общие определения суперблуждающих и равномерно суперблуждающих даны в [4]. Для краткости изложения дадим здесь упрощенное определение, используя тот факт, что в построенных примерах блуждающее множество двусвязно и гомеоморфно цилиндру, а сужение рассматриваемых отображений на этот цилиндр является локальным гомеоморфизмом.

Определение 3 Точка $x$ называется нейтралъно блуждающей точкой $f$, если найдется такая ее связная окрестность $U$, что $\forall n \geq 0$ открьтое множество $f^{-n}\left(f^{n}(U)\right)$ распадается на компоненты связности такие, что сужение $f$ на каждую компоненту связности является гомеоморфизмом и каждая компонента связности содержит в точности одно точку из множества $\left\{f^{-n}\left(f^{n}(x)\right)\right\}$.

Определение 4 Точка $x$ называется суперблуждающей точкой $f$, если она блуждающая и нейтрально блуждающая.

Обозначим через $\Omega$ множество неблуждающих (не являющихся блуждающими) точек.

Определение 5 Блуждающая точка $x$ называется регулярной, если $\forall \epsilon>$ $0 \exists \delta(x)-\delta$-окрестность точки $x, u \exists N>0$ такое, что $\forall k>N u \forall k<-N$ $f^{k}(\delta(x)) \subset \epsilon(\Omega)$, где $\epsilon(\Omega)-\epsilon$-окрестность множества $\Omega, f^{k}(\delta(x))-$ образ $\delta(x)$ при отображении $f^{k}$.

Множество регулярных точек открыто. Максимальные связные компоненты этого множества называются регулярными компонентами.

\section{Построение искомой последовательности.}

Введем на сфере без двух точек координаты бесконечного цилиндра $(r, \phi), r \in \mathbb{R}, \phi \in[0,1)$. Две оставшиеся точки сферы обозначим соответственно через $+\infty$ и $-\infty$.

Рассмотрим в этих координатах простое отображение сферы

$$
\Phi_{0}:(r, \phi) \mapsto(r+1,2 \phi \bmod 1) .
$$

Легко видеть, что это внутреннее отображение степени 2 с точками ветвления $+\infty$ и $-\infty$. Неблуждающее множество этого отображения тоже состоит 
из точек $+\infty$ и $-\infty$, при чем $+\infty$ является стоком, а $-\infty-$ источником. Остальные точки являются блуждающими, при чем блуждающее множество обладает двумя инвариантными слоениями на координатные линии $\{r=$ const $\}$ и $\{\phi=$ const $\}$.

Следующее семейство отображений будет построено с помощью возмущений $\Phi_{0}$, которые мы будем подбирать так, чтобы у возмущенного отображения неблуждающее множество продолжало бы состоять из точек ветвления $+\infty$ и $-\infty$, и сохранялись инвариантные слоения $\{r=$ const $\}$ и $\{\phi=$ const $\}$.

Для этого в отображении $\Phi_{0}$ будем менять во второй (угловой) координате отображение $\Psi_{0}=2 \phi \bmod 1$ на отображения $\Psi_{i}(\phi)$, где каждое $\Psi_{i}: S^{1} \rightarrow S^{1}$ - некоторое возмущение отображения $2 \phi \bmod 1$, также являющееся двулистным накрытием окружности $\mathbb{R} \bmod 1$, которое будет задано далее. Тогда искомое семейство отображений $\left(\Phi_{i}\right), i \geq 1$ будет иметь вид $\Phi_{i}:(r, \phi) \mapsto\left(r+1, \Psi_{i}(\phi)\right)$. Легко видеть, что для отображений такого вида слоения $\{r=$ const $\}$ и $\{\phi=$ const $\}$ являются инвариантными.

Осталось задать отображения $\left(\Psi_{i}\right), i \geq 1$. Заметим, что у отображения $\Psi_{0}=2 \phi \bmod 1$ множество периодических точек бесконечно и их максимальный период не ограничен. Выберем какую-нибудь бесконечную последовательность $\left(\varepsilon_{0}^{n}\right)$ периодических точек отображения $2 \phi \bmod 1$ такую, что период этих точек $p(n)$ строго возрастает. $\mathrm{K}$ примеру, положим $\varepsilon_{0}^{1}=\frac{1}{2}$ (период $\left.1, O_{\Psi_{0}}^{+}\left(\frac{1}{2}\right)=\left\{\frac{1}{2}\right\}\right), \varepsilon_{0}^{2}=\frac{1}{3}$ (период $\left.2, O_{\Psi_{0}}^{+}\left(\frac{1}{3}\right)=\left\{\frac{1}{3}, \frac{2}{3}\right\}\right), \varepsilon_{0}^{3}=\frac{1}{7}$ (период 3 , $\left.O_{\Psi_{0}}^{+}\left(\frac{1}{7}\right)=\left\{\frac{1}{7}, \frac{2}{7}, \frac{4}{7}\right\}\right), \varepsilon_{0}^{4}=\frac{1}{5}$ (период $\left.4, O_{\Psi_{0}}^{+}\left(\frac{1}{5}\right)=\left\{\frac{1}{5}, \frac{2}{5}, \frac{4}{5}, \frac{3}{5}\right\}\right), \ldots$

Обозначим точки из $O_{\Psi_{0}}^{+}\left(\varepsilon_{0}^{n}\right)$ через $\varepsilon_{0}^{n}, \ldots, \varepsilon_{p(n)-1}^{n}, \Psi_{0}\left(\varepsilon_{i}^{n}\right)=\varepsilon_{i+1}^{n}$. Пусть $d_{n}=\min _{i, j}\left|\varepsilon_{i}^{n}-\varepsilon_{j}^{n} \bmod 1\right|-$ минимальное расстояние между точками из $O^{+}\left(\varepsilon_{0}^{n}\right)$. Обозначим через $I_{j}^{i}$ отрезок $\left[\varepsilon_{j}^{i}-\frac{d_{i}}{4}, \varepsilon_{j}^{i}+\frac{d_{i}}{4}\right]$.

Зададим $\Psi_{i}(\phi)$ на отрезках $I_{j}^{i}$ как $\Psi_{i}(\phi)=\varepsilon_{j}^{i}+\phi, \phi \in I_{j}^{i}$, а вне отрезков $I_{j}^{i}$ гладко продолжим $\Psi_{i}(\phi)$ любой подходящей функцией так, чтобы отображение $\Psi_{i}(\phi)$ было монотонно по $\phi$ и вне отрезков $I_{j}^{i}$ производная от $\Psi_{i}(\phi)$ была бы строго больше 1 . Заметим, что по построению $\Psi_{i}\left(I_{j}^{i}\right)=I_{j+1}^{i}$, $j<p(n)-1, \Psi_{i}\left(I_{p(n)-1}^{i}\right)=I_{0}^{i}, I_{p(n)}^{i}=I_{0}^{i}$. K примеру, в качестве $\Psi_{i}$ можно взять функции из рис. 1 . Динамика $\Psi_{i}(\phi)$ выгдядит следующим образом: по построению, отрезки $I_{j}^{i}$ состоят из периодических точек с периодом $2^{i}$. Оставшиеся точки принадлежат либо открытому множеству точек, которые отображаются на внутренность отрезков $I_{j}^{i}$, либо входят в оставшееся Канторово множество растягивающих точек, состоящее из счетного множества 
нестрого растягивающих точек, входящих в границы прообразов отрезков, и континуума строго растягивающих точек.
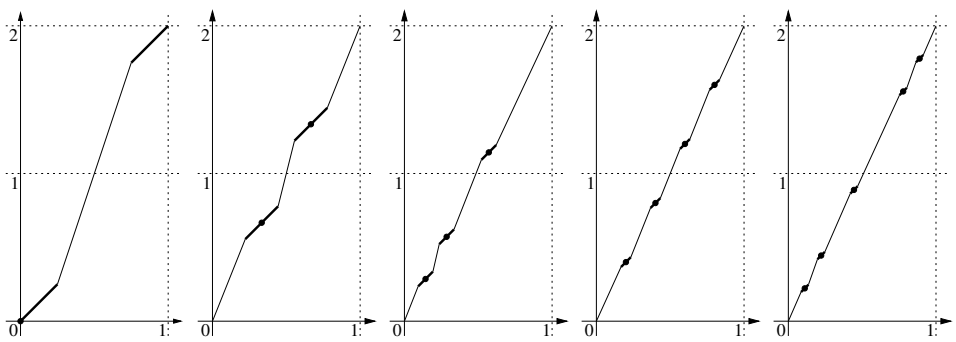

Рис. $1 \Psi_{1}(\phi), \Psi_{2}(\phi), \Psi_{3}(\phi), \Psi_{4}(\phi), \Psi_{5}(\phi)$.

Рассмотрим у полученного внутреннего отображения $\Phi_{i}$ произвольную блуждающую точку $\left(r_{0}, \phi_{0}\right)$. Представим широкую траекторию точки $\left(r_{0}, \phi_{0}\right)$ в виде объединения нейтральных сечений:

$$
O_{\Phi_{i}}\left(\left(r_{0}, \phi_{0}\right)\right)=\cup_{s=-\infty}^{+\infty}\left(\Phi_{i}\right)^{s}\left(O_{\Phi_{i}}^{\perp}\left(r_{0}, \phi_{0}\right)\right) .
$$

По построению, ее широкая траектория вложена в набор окружностей

$$
\left\{\left\{r_{0}+n=\text { const }\right\}, n \in \mathbb{Z}\right\},
$$

а каждое нейтральное сечение $\left(\Phi_{i}\right)^{s}\left(O_{\Phi_{i}}^{\perp}\left(r_{0}, \phi_{0}\right)\right)$ широкой траектории лежит в своей отдельной окружности $\left\{r_{0}+s=c o n s t\right\}$, и, как следствие, разные нейтральные сечения отделены друг от друга. Отсюда следует, что является ли точка $\left(r_{0}, \phi_{0}\right)$ просто блуждающей или же суперблуждающей, зависит только от ее динамики по угловой координате $\phi$, заданной отображением $\Psi_{i}(\phi)$.

Легко видеть, что точки, для которых их угловая координата со временем отображается с помощью $\Psi_{i}(\phi)$ на внутренность одного из отрезков $I_{j}^{i}$, являются суперблуждающими. В качестве разделяющей окрестности из определения там можно взять внутренность прямоугольника $I_{j}^{i} \times\left(r_{0}-\varepsilon, r_{0}+\right.$ $\varepsilon)$. Оставшиеся точки не являются суперблуждающими, так как по построению они являются точками накопления своего нейтрального сечения.

Таким образом, для отображения $\Phi_{i}$ отрезки $I_{j}^{i}$ однозначно определяют вперед инвариантные компоненты множества суперблуждающих точек.

В полученном семействе отображений $\left(\Phi_{i}\right), i \geq 1$ вперед инвариантные периодические компоненты множества суперблуждающих точек отображения $\Phi_{i}$ по построению представляют собой внутренности множеств 
$I_{j}^{i} \times(-\infty,+\infty)$ и имеют период $p(i)$. Поскольку период $p(i)$ растет строго монотонно, для разных $i$ отображения $\Phi_{i}$ не могут быть топологически сопряжены между собой, а их множества суперблуждающих точек топологически различны.

\section{Список литературы}

1. Birkhoff, G., Smith, P.: Structure analysis of surface transformations. J. Math. 7, 357-369 (1928).

2. Smith, P.: The Regular Components of Surface Transformations. Amer. Jour. of math., Baltimore. 52, No. 2, 357-369 (1930).

3. Арансон, С. Х., Медведев, В. С.: Регулярные компоненты гомеоморфизмов $n$-мерной сферы. Мат. Сборник. 85(127), №1(5), 3-17 (1971).

4. Власенко И. Ю.: Внутренние отображения: топологические инварианты и их приложения. Праці Інституту математики НАН України. Математика та ї̈ застосування. Том 101. Институт математики НАН Украины. Киев, 2014, 225 стр.

5. Кузаконь, В. М., Кириченко, В. Ф., Пришляк, О. О.: Гладкі многовиди: геометричні та топологічні аспекти. Праці Інституту математики НАН України. Математика та ï̈ застосування. Том 97, Институт математики НАН Украины, Киев, 2013, 500 стр.

6. Стоилов, С.: О топологических принципах теории аналитических функций. М., Мир. 1964, 228 стр.

7. Трохимчук, Ю. Ю.: Дифференцирование, внутренние отображения и критерии аналитичности. Институт математики НАН Украины. Киев, 2008, 538 стр.

\section{Игорь Юрьевич Власенко}

Институт математики НАНУ, Киев, Украина

E-mail: vlasenko@imath.kiev.ua

\section{Igor Yu. Vlasenko}

\section{Abundance of topological conjugacy classes for wandering sets of inner mappings}

For a homeomorphism there is only one cylindric regular invariant component of wandering set up to the topological conjugacy. But it is different for inner mappings. This paper shows an example of infinite sequence of inner mappings such that each of them has a cylindric regular invariant component of wandering set but for any pair of inner mappings their components are not topologically conjugate. 